\title{
A reconfigurable software defined ultra-wideband impulse radio transceiver
}

\author{
M. D. Blech ${ }^{1}$, A. T. Ott ${ }^{2}$, P. Neumeier ${ }^{3}$, M. Möller ${ }^{1}$, and T. F. Eibert ${ }^{2}$ \\ ${ }^{1}$ Institut für Hochfrequenztechnik, Universität Stuttgart, 70550 Stuttgart, Germany \\ ${ }^{2}$ Lehrstuhl für Hochfrequenztechnik, Technische Universität München, 80290 Munich, Germany \\ ${ }^{3}$ Abteilung Leistungsoptimierte Systeme, Fraunhofer-Institut für Integrierte Schaltungen IIS, 90411 Nürnberg, Germany
}

\begin{abstract}
An ultra-wideband (UWB) software defined radio (SDR) implementation is presented. The developed impulse radio (IR) transceiver employs first order bandpass (BP) sampling at a conversion frequency which is four times the channel bandwidth. The subsampling architecture directly provides the RF signal avoiding any non-ideal mixer stages and reduces the requirements of digital signal processing implemented in a field programmable gate array (FPGA). The transmitter consists basically of a multi-Nyquist digital to analog converter (DAC), whereas the implemented matched filter (MF) receiver prototype employs a standard digitizing oscilloscope. This design can be adaptively reconfigured in terms of modulation, data rate, and channel equalization. The reconfigurable design is used for an extensive performance analysis of the quadrature phase shift keying (QPSK) modulation scheme investigating the influence of different antennas, amplifiers, narrowband interferers as well as different equalizer lengths. Even for distances up to $7 \mathrm{~m}$ in a multipath environment robust communication was achieved.
\end{abstract}

\section{Introduction}

The approval of UWB technology in the US and in Europe has caused enormous research activities on broadband wireless communication schemes (Reed, 2005). Up to now mainly solutions based on orthogonal frequency division multiplexing (OFDM) (Batra et al., 2004) or direct sequence spread spectrum (DSSS) (Fisher et al., 2004) are commercially available. IR devices are still a niche product (Time Domain Corporation, 2006).

An SDR concept offers the best opportunities to combine the advantages of impulse based UWB communication with the flexibility required by modern transceiver designs. Re-

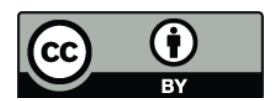

Correspondence to: M. D. Blech (mail@ihf.uni-stuttgart.de) cently, some investigations dealing with UWB SDR concepts have been published (Chen and Brodersen, 2007; Keller et al., 2007) mainly focusing on the receiver implementation and using analog pulse generators on the transmitter side.

The flexibility of an UWB SDR transceiver implementation must be gained by a huge computational effort necessary for the wideband digital signal processing of a bandwidth in excess of $500 \mathrm{MHz}$ as required by the regulation. In ordinary SDR designs the signal on an intermediate frequency (IF) is digitized whereas up- and downconversion to the radio frequency (RF) band afford additional analog mixer stages (see Fig. 1 top) causing signal distortions due to their non-ideal transfer characteristic. Employing bandpass sampling (Vaughan et al., 1991) a subsampling direct conversion transceiver can be implemented avoiding any analog circuitry except for bandpass filters and optional amplifiers (see Fig. 1 bottom). First order sampling on the one hand relaxes the requirements of the DAC stage, but on the other hand limits the usable frequency range (Vaughan et al., 1991). Second order sampling offers more advantages in terms of frequency agility, but in turn requires more complex digital signal processing (Coulson et al., 1994). The application of either of these concepts is difficult in narrowband communications because of noise aliasing, but in UWB systems the performance degradation due to noise folding is negligible (Chen and Brodersen, 2007).

Thus, a novel subsampling UWB direct conversion SDR IR transceiver is presented. This reconfigurable prototype is designed for laboratory use for the analysis of various UWB IR communication schemes. Its transmitter basically consists of a field programmable gate array (FPGA) (Avnet, Inc., 2006) and a multi-Nyquist DAC (Maxim Integrated Products, Inc., 2006) enabling adaptive predistortion to equalize the channel impulse response, whereas a commercial digitizing oscilloscope is used on the receiver side of the transceiver prototype. Signal processing of the receiver is carried out offline, but with full applicability in real-time on adequate hardware. The trend of semiconductor technology towards

Published by Copernicus Publications on behalf of the URSI Landesausschuss in der Bundesrepublik Deutschland e.V. 

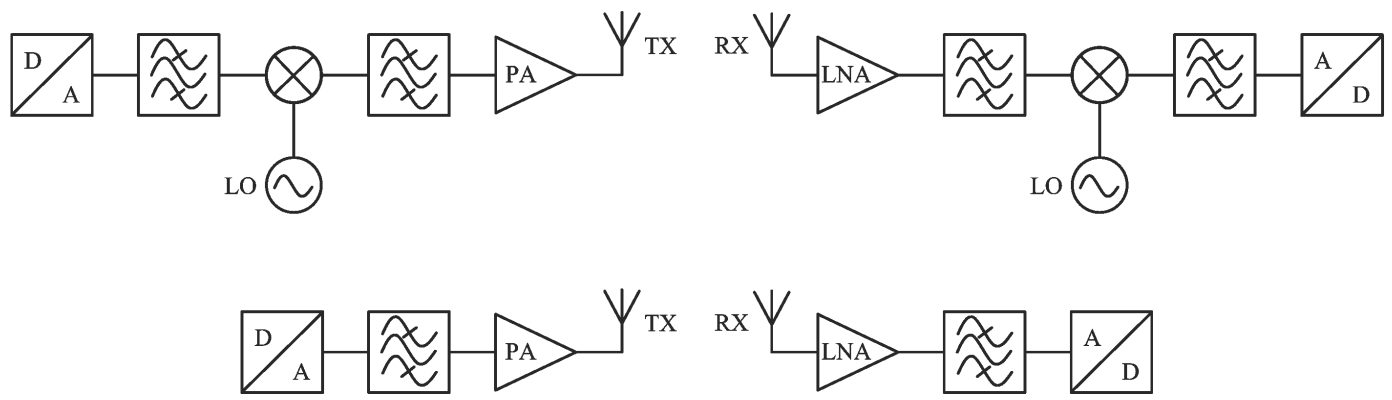

Fig. 1. Block diagram of a conventional SDR transceiver (top) and a subsampling architecture (bottom).
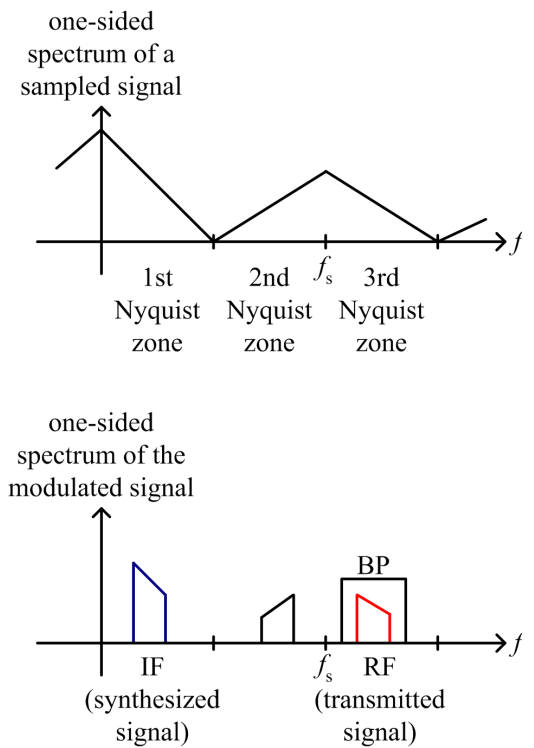

Fig. 2. Spectrum of a time discrete signal (top) and spectrum of a band-limited modulated signal (bottom).

higher frequencies and lower power consumption will make UWB SDR concepts applicable in consumer products in the near future.

In this paper the transceiver architecture of the developed prototype is described in Sect. 2. In Sect. 3 the performance of the SDR employing pulsed QPSK modulation is analyzed by numerical simulations and by measurements. Finally the work is concluded in Sect. 4.

\section{SDR transceiver}

The developed transceiver uses the subsampling architecture depicted in Fig. 1 (bottom). It uses first order sampling and is designed for the RF frequency range of $2-3 \mathrm{GHz}$, which is outside the approved UWB frequency band, but adequate to investigate the basic properties of an UWB communication system in a laboratory environment.

The sampling frequency $f_{\mathrm{s}}$ of both the DAC in the transmitter and the $\mathrm{ADC}$ in the receiver is $2 \mathrm{GHz}$, enabling the

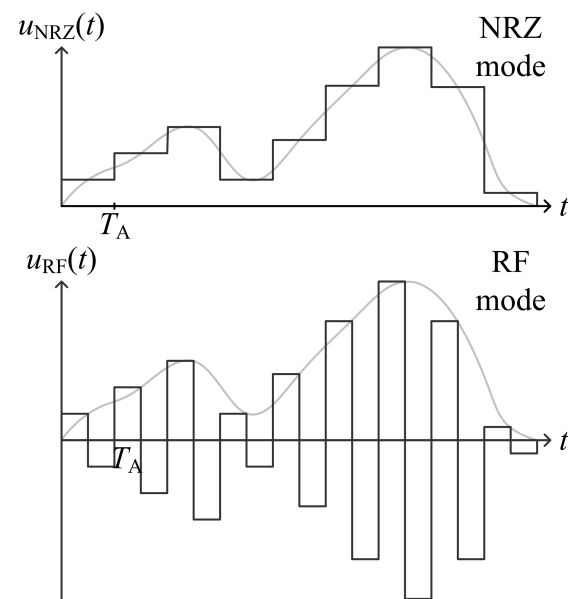

Fig. 3. DAC output signals (black) of a digitized analog waveform (gray) in different modes.

transmission of impulses exhibiting a bandwidth of up to $1 \mathrm{GHz}$. The employed QPSK modulation scheme uses Gaussian impulses as described in (Blech et al., 2008) with a $-10 \mathrm{~dB}$ bandwidth of $500 \mathrm{MHz}$ and a pulse repetition frequency $(\mathrm{PRF}) f_{\text {sym }}=0.5 \ldots 62.5 \mathrm{MHz}$.

\subsection{Transmitter architecture}

Each time discrete signal has a periodic spectrum as shown in Fig. 2 (top). Synthesizing a signal on an intermediate frequency (IF) in the first Nyquist zone does also generate multiple attenuated replicas in the higher Nyquist zones (see Fig. 2 bottom). Using an anti-aliasing filter exhibiting a BP instead of a lowpass (LP) characteristic directly provides the desired RF signal.

The employed DAC offers different modes, namely the non return to zero (NRZ), the return to zero (RZ), and the so called RF mode. Each of them is dedicated to applications in different Nyquist zones. The according output signals of an exemplary waveform in all aforementioned modes are depicted in Fig. 3. 
In the NRZ mode, which is usually used for LP signals, each step of the sampled waveform is described by a rectangular impulse

$h_{\mathrm{NRZ}}(t)=\operatorname{rect}\left(\frac{t}{T_{\mathrm{S}}}\right) \bullet \cdot T_{\mathrm{S}} \operatorname{sinc}\left(\pi f T_{\mathrm{S}}\right)=H_{\mathrm{NRZ}}(f)$,

where $T_{\mathrm{S}}$ denotes the sampling interval, $h_{\mathrm{NRZ}}(t)$ is the time domain waveform, and $H_{\mathrm{NRZ}}(f)$ stands for the frequency domain representation of one impulse.

The same holds for the bipolar RF impulse

$$
\begin{aligned}
h_{\mathrm{RF}}(t) & =\operatorname{rect}\left(\frac{t+\frac{T_{\mathrm{s}}}{4}}{\frac{T_{\mathrm{s}}}{2}}\right)-\operatorname{rect}\left(\frac{t-\frac{T_{\mathrm{s}}}{4}}{\frac{T_{\mathrm{s}}}{2}}\right) \\
& \bullet \mathrm{j} T_{\mathrm{s}} \frac{1-\cos \left(\pi f T_{\mathrm{S}}\right)}{\pi f T_{\mathrm{s}}}=H_{\mathrm{RF}}(f),
\end{aligned}
$$

where the impulse is bisected, has no direct current (DC) component and exhibits higher spectral amplitudes in the 2nd and 3rd Nyquist zone compared to the NRZ mode (Maxim Integrated Products, Inc., 2006).

The pulse train to be transmitted is composed of analog impulses $g(t)$ (Blech et al., 2008), which are sampled at the sampling interval $T_{\mathrm{S}}$ yielding the signal

$g_{\mathrm{s}}(t)=g(t) \sum_{n=-\infty}^{\infty} \delta\left(t-n T_{\mathrm{S}}\right)=\sum_{n=-\infty}^{\infty} g\left(n T_{\mathrm{s}}\right) \delta\left(t-n T_{\mathrm{s}}\right)$

In order to obtain the spectrum of the DAC output signal $g_{\text {DAC }}(t)$, the sampled pulse train must be convolved with the corresponding impulse waveform of the chosen DAC mode

$g_{\mathrm{DAC}}(t)=h(t) * g_{\mathrm{s}}(t)$.

This yields the output spectra

$$
\begin{gathered}
G_{\mathrm{s}, \mathrm{NRZ}}(f)=H_{\mathrm{NRZ}}(f) \frac{1}{T_{\mathrm{s}}} \sum_{k=-\infty}^{\infty} G_{\mathrm{s}}\left(f-\frac{k}{T_{\mathrm{s}}}\right), \\
G_{\mathrm{s}, \mathrm{RF}}(f)=H_{\mathrm{RF}}(f) \frac{1}{T_{\mathrm{s}}} \sum_{k=-\infty}^{\infty} G_{\mathrm{s}}\left(f-\frac{k}{T_{\mathrm{s}}}\right) .
\end{gathered}
$$

In Fig. 4 the measured output spectra of the NRZ and RF mode are depicted. The signal is measured directly at the output of the DAC board synthesizing a Gaussian shaped impulse train with full scale amplitude. The RF mode provides the highest output amplitude in the third Nyquist zone and is used in this work. There the output signal needs further amplification to exploit the maximum permitted power spectral density (PSD) of $-41.3 \mathrm{dBm} / \mathrm{MHz}$.

The PSD of a modulated pulse train analytically results in

$$
\begin{aligned}
\operatorname{PSD}(f) & =\frac{\sigma_{\mathrm{b}}^{2}}{T_{\mathrm{sym}}} \frac{\left|G_{\mathrm{s}, \mathrm{RF}}(f)\right|^{2}}{Z_{\mathrm{c}}} \\
& +\frac{\mu_{\mathrm{b}}^{2}}{T_{\mathrm{sym}}^{2}} \sum_{m=-\infty}^{\infty} \frac{\left|G_{\mathrm{s}, \mathrm{RF}}(f)\right|^{2}}{Z_{\mathrm{c}}} \delta\left(f-\frac{m}{T_{\mathrm{sym}}}\right)
\end{aligned}
$$

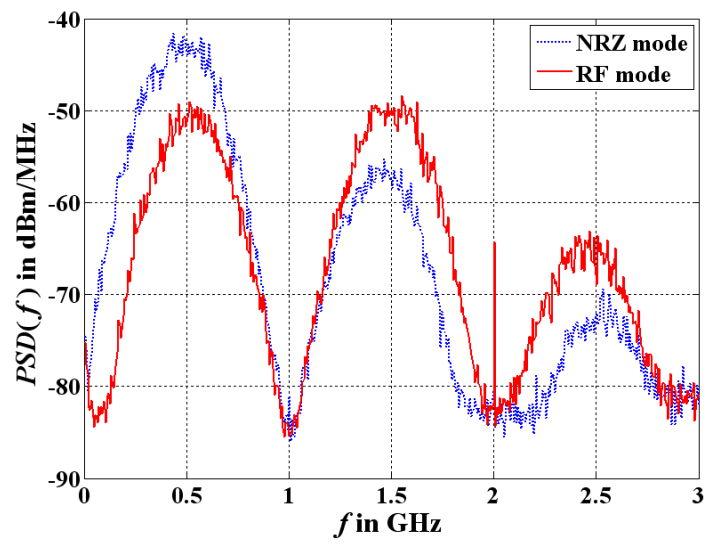

Fig. 4. Measured PSD of the QPSK output signal in different DAC modes $\left(f_{\mathrm{s}}=2 \mathrm{GHz}, f_{\mathrm{sym}}=62.5 \mathrm{MHz}, f_{\mathrm{c}}=500 \mathrm{MHz}, B=500 \mathrm{MHz}\right)$.

for an equidistant sequence of symbols $b$ spaced by $T_{\text {sym }}$ (Proakis, 2001). In Eq. (7) $Z_{\mathrm{C}}$ stands for the characteristic impedance, in this case $50 \Omega$, whereas $\mu_{\mathrm{b}}$ and $\sigma_{\mathrm{b}}^{2}$ denote the expected value and the variance of the transmitted symbols, respectively. As a pseudo random binary sequence (PRBS) generator is used $\mu_{\mathrm{b}}=0$ and the second part of the sum vanishes.

In order to filter the desired RF signal in the third Nyquist zone a substrate integrated waveguide (SIW) filter (Nam et al., 2005) is used to suppress spectral components in the first and second Nyquist zone and to avoid aliasing. This filter exhibits a highpass (HP) characteristic with an almost constant group delay and with a cut-off frequency of $2.15 \mathrm{GHz}$ as well as a rejection of more than $40 \mathrm{~dB}$ below $2 \mathrm{GHz}$. Due to the limited rise time of the output signal of the DAC and the LP characteristic of the output network on the DAC board, spectral components in the Nyquist zones four or higher do actually not exist and thus need not to be filtered explicitly. However, amplification is necessary.

The very high sampling frequency of $2 \mathrm{GHz}$ requires an immense parallelization of the transmitter design in the FPGA implementation. The 12 bit DAC already includes a 4:1 multiplexer splitting up the data bus in four branches (AD), each consisting of 12 low voltage differential signaling (LVDS) lines. But as the Virtex-5 FPGA from Xilinx (Avnet, Inc., 2006) must meet some timing constraints, another 2:1 multiplexer consisting of the internal output serializer deserializer (OSERDES) blocks is necessary reducing the FPGA clock to $f_{\mathrm{s}} / 8=250 \mathrm{MHz}$. Each QPSK waveform is stored in two random access memory (RAM) clusters (Fig. 5 left), whose data is initialized via a universal serial bus (USB) interface by a host PC. The programmed waveform of arbitrary shape and user-defined length $N$ is directly linked to the symbol clock frequency $f_{\text {sym }}$. The according clock signals and the composition of a waveform are plotted in Fig. 5 (right). As shown in the diagram, Gray-coding is used. Though using only 11 of the 12 differential data lines of each data bus, 


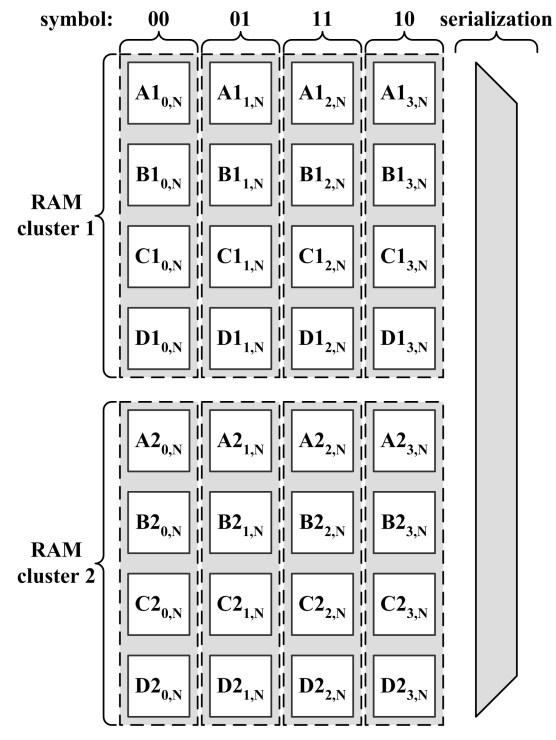

Fig. 5. Memory organization of the transmit symbols (left) and according transmit waveform (right).

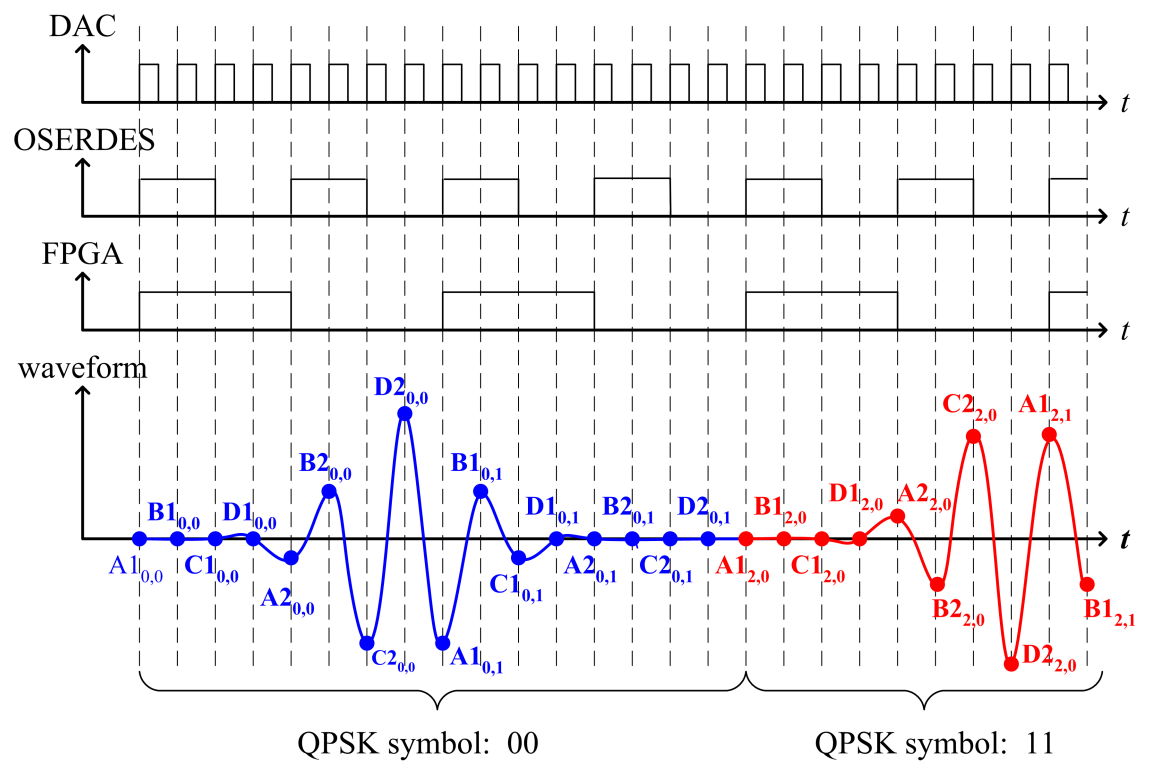

still 88 lines between FPGA and DAC and an update rate of $22 \mathrm{Gbit} / \mathrm{s}$ are required.

\subsection{Receiver design}

A correlation receiver is implemented in MATLAB for offline use as well as for real-time reception in Handel-C for an FPGA design. The receiver uses the digitized waveform

$v_{\mathrm{S}, \mathrm{RX}}(t)=v_{\mathrm{RX}}(t) \sum_{n=-\infty}^{\infty} \delta\left(t-n T_{\mathrm{S}}\right)$

obtained after sampling the analog receive signal $v_{\mathrm{RX}}(t)$. The according spectrum

$V_{\mathrm{s}, \mathrm{RX}}(f)=V_{\mathrm{RX}}(f) * \frac{1}{T_{\mathrm{s}}} \sum_{k=-\infty}^{\infty} \delta\left(f-\frac{k}{T_{\mathrm{s}}}\right)$

is periodic and a simple LP filter provides the desired replica in the first Nyquist zone. In the receiver prototype the receive signal is acquired by a real-time oscilloscope DSO81004A from Agilent running at $2 \mathrm{GS} / \mathrm{s}$. The RF frontend only consists of a BP filter (Mini-Circuits, 2008) and an optional low noise amplifier (LNA) with a gain of $48 \mathrm{~dB}$ and a noise figure of $1.6 \mathrm{~dB}$.

A block diagram of the MF receiver is given in Fig. 6. It demodulates the acquired receive signal $v_{\mathrm{RX}}(t)$ offline, but it meets the requirements for a real-time application on e.g. an FPGA running at $f_{\mathrm{s}} / 4$. All filters are of finite impulse response (FIR) type, which is advantageous for parallelization.

The equalizer impulse response $h_{\text {equ }}(t)$ is estimated using a preamble of up to 100 symbols and a time domain deconvolution technique (Preis, 1977) to determine the channel impulse response. In the QPSK modulation scheme four matched filters $\left(h_{\mathrm{MF}, 00}, \ldots, h_{\mathrm{MF}, 11}\right)$ and a subsequent maximum likelihood (ML) decision provide the digital receive symbols $\hat{b}$.

A portation for an FPGA based receiver has been carried out already, but due to the lack of an appropriate wideband $\mathrm{ADC}$ all results presented in this paper have been computed offline employing the aforementioned oscilloscope for the signal acquisition and the equivalent MATLAB implementation of the receiver.

\section{Performance analysis}

A complete model of the transceiver has been implemented in MATLAB in order to compare the simulated and measured system performance. In this paper exemplary results of a line-of-sight (LOS) QPSK IR communication in a distance ranging from 1 to $7 \mathrm{~m}$ are presented. In this investigation a symbol frequency $f_{\text {sym }}=62.5 \mathrm{MHz}$, a center frequency $f_{\mathrm{c}}=$ $2.5 \mathrm{GHz}$, and a bandwidth $B=500 \mathrm{MHz}$ are used.

\subsection{Simulations}

In the simulations the transmit waveform is generated taking into account the jitter affecting the transmit signal. However, the jitter of the commercial real-time oscilloscope on the receiver side is negligible. In UWB applications the impulse response of an antenna is an important issue. Thus the normalized impulse response (Farr and Baum, 1998) of the various investigated UWB antennas is computed using CST Microwave Studio. From the transfer functions $H_{\mathrm{N}, \mathrm{TX}}$ and 


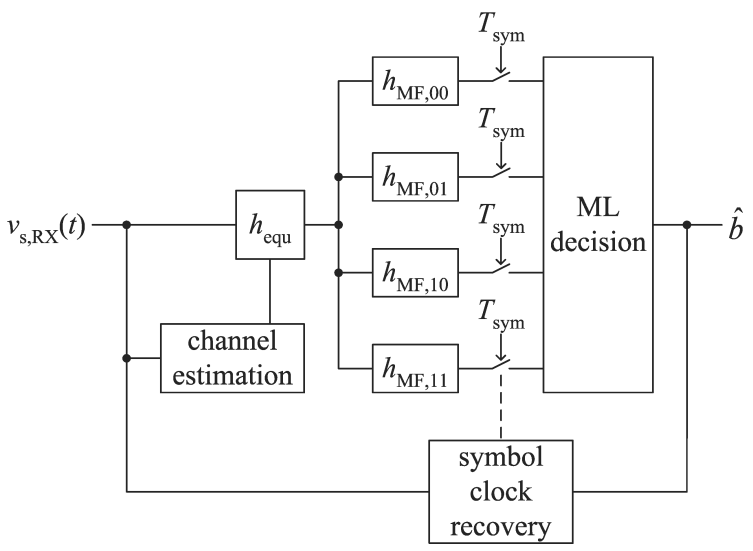

Fig. 6. Block diagram of the implemented matched filter receiver.

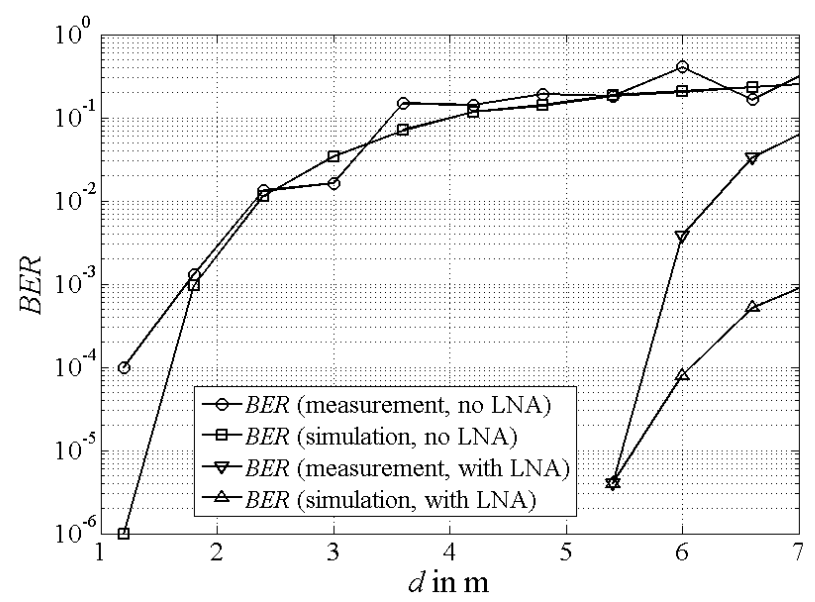

Fig. 7. Influence of the LNA on the simulated and measured BER for a LOS scenario employing directional vivaldi antennas.

$H_{\mathrm{N}, \mathrm{RX}}$ of the transmit and receive antenna, respectively, the modified "new" normalized transfer functions

$H_{\mathrm{N}, \text { new }, \mathrm{TX}}(\omega)=\mathrm{j} \omega \frac{1}{\mathrm{c}_{0}} \sqrt{\frac{\omega}{\pi}} H_{\mathrm{N}, \mathrm{TX}}(\omega)$

and

$H_{\mathrm{N}, \text { new }, \mathrm{RX}}(\omega)=\frac{1}{\mathrm{c}_{0}} \sqrt{\frac{\omega}{\pi}} H_{\mathrm{N}, \mathrm{RX}}(\omega)$

are derived according to Ott et al. (2009). Regarding the entire radio link, this enables the extraction of the channel transfer function $H_{\mathrm{CH}}$ from the antenna transfer characteristic defined in (Farr and Baum, 1998).

Thus the transmission parameter between transmitter and receiver can now be written as

$S_{21}(\omega)=H_{\mathrm{N}, \text { new }, \mathrm{TX}}(\omega) H_{\mathrm{CH}}(\omega) H_{\mathrm{N}, \text { new }, \mathrm{RX}}(\omega)$.

Knowing the characteristics of the transmit and receive antennas the channel impulse response is synthesized using the

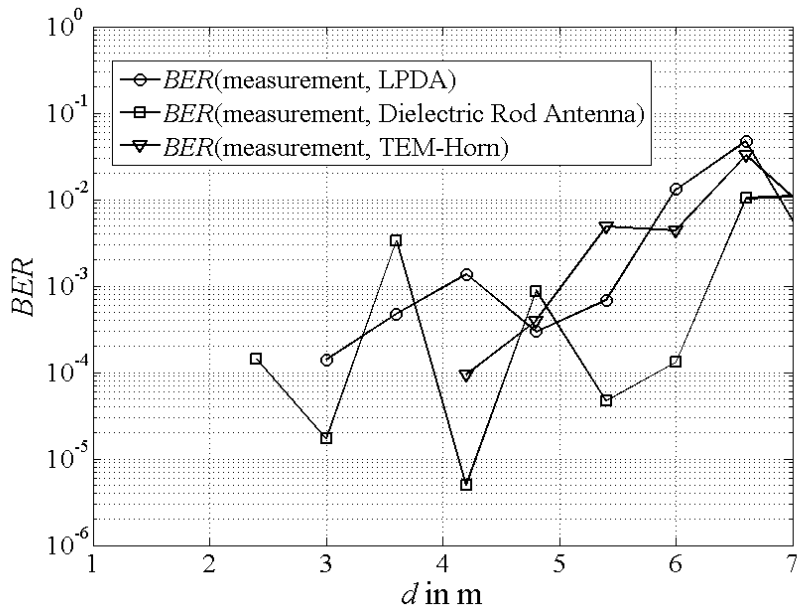

Fig. 8. Simulated and measured BER for a LOS scenario using different types of directional antennas.

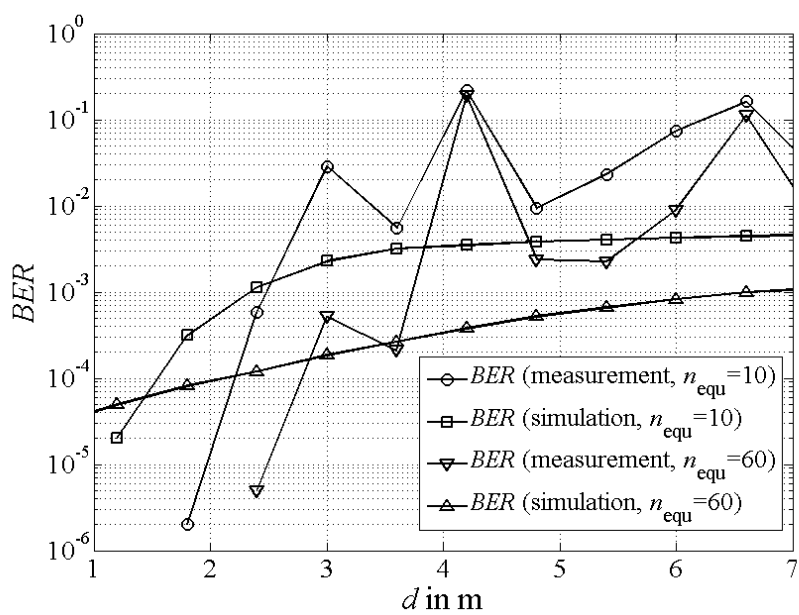

Fig. 9. Simulated and measured BER for a LOS scenario using omnidirectional biconical antennas and different equalizer lengths.

empirical UWB channel model presented in (Ghassemzadeh et al., 2005). For this purpose the parameters representing the environment at the authors' institute have been determined by a measurement campaign (Ott et al., 2009).

The receive voltage $v_{\mathrm{RX}}(t)$ is obtained by convolving all impulse responses of all individual components of the transfer chain. The demodulation is accomplished by the MF receiver implementation described in Sect. 2.2.

\subsection{Measurements}

Measurements in one exemplary test site have been carried out using the SDR IR transceiver described in Sect. 2. The receive signal is acquired by a digitizing oscilloscope DSO81004A from Agilent, which can record up to $1 \mathrm{MSym}$ bols. For the demodulation and the evaluation of the BER the same receiver program is used as in the simulation. 


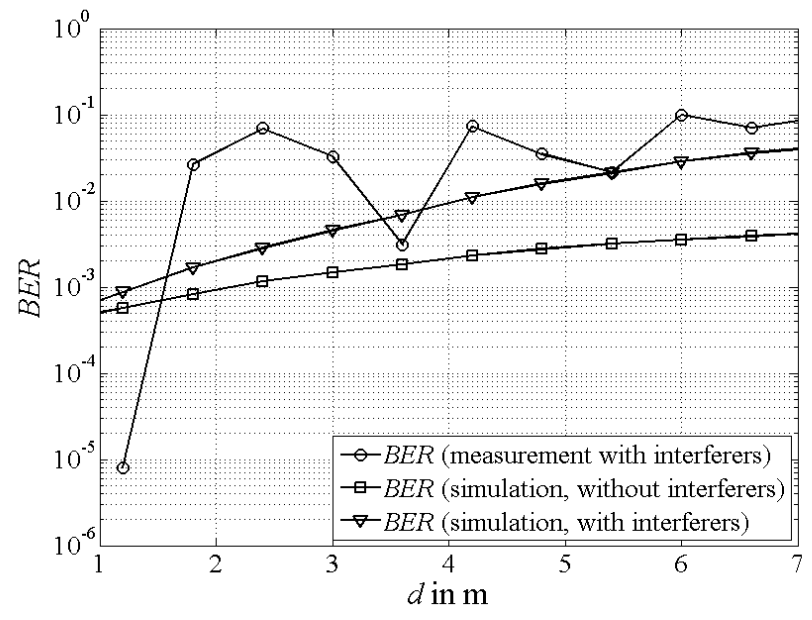

Fig. 10. Comparison of the measured and simulated BER for a LOS scenario with and without interferers using omnidirectional biconical antennas.

In Fig. 7 the measured BER achieved with two directive Vivaldi antennas pointing straight from the transmitter to the receiver is presented. The measurements are compared to simulation results obtained for an ideal LOS channel. It can be seen that the optional LNA can improve the BER significantly, whereas the imperfections of the channel are negligible.

Furthermore different types of highly directive antennas are used to measure the BER. The antennas can be divided in two classes: On the one hand a logarithmic periodic dipole antenna (LPDA) which suffers from dispersion and on the other hand antennas for impulse radio applications like the TEM horn or the dielectric rod antenna presented by (Blech and Eibert, 2007), which all exhibit an excellent transient behavior, are considered. As depicted in Fig. 8 a low BER is achieved with all types of antennas which means that the influence of the antennas can be compensated by the equalizer of the correlation receiver.

Figure 9 shows a LOS scenario of two omnidirectional biconical antennas which are more sensitive to multipath propagation. In this comparison the LNA has always been used and the simulation results showing the average BER have been computed using the channel model of (Ghassemzadeh et al., 2005). The number of taps $n_{\text {equ }}$ of the FIR equalizer $h_{\text {equ }}$ is varied. As depicted in Fig. 9 a longer filter can slightly improve the BER as inter symbol interference (ISI) can be compensated in addition.

Comparing the measured BER in Fig. 10 in an commercial building with an ideal simulation, strong deviations can be seen. By considering the influence of the Wi-Fi network in the system simulation a much better correspondence to the measurement is achieved. Thus narrowband interferers have an impact on the signal quality but they do not completely corrupt the UWB signal.

\section{Conclusions}

A prototype of a flexible subsampling UWB software defined IR transceiver has been presented. It can be reconfigured and used for the analysis of the performance of various IR modulation schemes such as e.g. QPSK, which is investigated in this paper. The design uses first order BP sampling avoiding any non-ideal mixer stages. As numerical system simulations and practical measurements of the BER in an exemplary environment show, the presented IR concept is a real alternative to OFDM systems offering the speed and radio link quality demanded by modern wireless UWB communications.

\section{References}

Avnet, Inc.: Xilinx Virtex-5 LX50 Development Kit, http://www. avnet.com, 2006.

Batra, A., and 78 co-authors: IEEE Multi-band OFDM Physical Layer Proposal for IEEE 802.15 Task Group 3a, 2004.

Blech, M. D. and Eibert, T. F. : A Dipole Excited Ultrawideband Dielectric Rod Antenna With Reflector, IEEE Transactions on Antennas and Propagation, 55, 1948-1954, 2007.

Blech, M. D., Geier, D., and Eibert, T. F.: Concept of an UWB Impulse Radio B-/QPSK Transmitter Based on Standard Logic Components, in: Proc. IEEE ICUWB 2008, pp. 225-228, Hannover, Germany, 2008.

Coulson, A. J., Vaughan, R. G., and Poletti, M. A.: FrequencyShifting Using Bandpass Sampling, IEEE Transactions on Signal Processing, 42, 1556-1559, 1994.

Chen, S.-W. M. and Brodersen, R. W.: A Subsampling Radio Architecture for Ultrawideband Communications, IEEE Transactions on Signal Processing, 55, 5018-5031, 2007.

Farr, E. G. and Baum, C. E.: Time Domain Characterization of Antennas with TEM Feeds, http://www.farr-research.com/Papers/ ssn426.pdf, 1998.

Fisher, R., Kohno, R., McLaughlin, M., and Welborn, M.: DSUWB Physical Layer Submission to 802.15 Task Group 3a, 2004.

Ghassemzadeh, S. S., Greenstein, L. J., Sveinsson, T., Kavcic, A., and Tarokh, V.: UWB Delay Profile Models for Residential and Commercial Indoor Environments, IEEE Transactions on Vehicular Technology, 54, 1235-1243, 2005.

Keller, C. M., Burkhart, J. M., and Phuong, T. T.: Ultra-Wideband Direct Sampling Receiver, in: Proc. IEEE ICUWB 2007, pp. 387-392, Singapore, Singapore, 2007.

Maxim Integrated Products, Inc.: 12-Bit, 2.3Gsps, MultiNyquist DAC with Selectable Frequency Response, http://www. maxim-ic.com, 2006.

Mini-Circuits: Coaxial Bandpass Filter VBFZ-2575+, http://www. mini-circuits.com, 2008.

Nam, H., Yun, T.-S., Kim, K.-B., Yoon, K.-C., and Lee, J.-C.: KuBand Transition Between Microstrip and Substrate Integrated Waveguide (SIW), in: Proc. IEEE APMC 2005, vol. 1, Suzhou, China, 2005.

Ott, A. T., Blech, M. D., Kraemer, M., and Eibert, T. F.: Enhanced Parameter Determination of an Empirical UWB Channel Model for European Building Standards, in: Proc. GeMiC 2009, Munich, Germany, 2009. 
Preis, D.: Least-Squares Time-Domain Deconvolution for Transversal-Filter Equalisers, IEEE Electronics Lett., 13, 356357, 1977.

Proakis, J. G.: Digital Communications, McGraw-Hill, New York, NY, 4th edn., 2001.

Reed, J. H.: An Introduction to Ultra Wideband Communication Systems, Prentice Hall, Upper Saddle River, NJ, 2005.
Time Domain Corporation: PulsOn P210 Evaluation Kit, http: //www.timedomain.com, 2006.

Vaughan, R. G., Scott, N. L., and White, D. R.: The Theory of Bandpass Sampling, IEEE Transactions on Signal Processing, 39, 1973-1984, 1991. 